\title{
CENTRAL MEASURES ON SEMISIMPLE LIE GROUPS HAVE ESSENTIALLY COMPACT SUPPORT
}

\author{
DAVID L. RAGOZIN ${ }^{1}$ AND LINDA PREISS ROTHSCHILD
}

\begin{abstract}
In this paper it is shown that for a connected semisimple Lie group with no nontrivial compact quotient any finite central measure is a discrete measure concentrated on the center of the group. More generally, the largest possible support set for a central measure on any semisimple Lie group is determined. From these results it follows that the center of the algebra $L_{1}(H)$ is trivial for any locally compact group $H$ which has a noncompact connected simple Lie group as a homomorphic image.
\end{abstract}

Let $\mu$ be a finite complex measure on a locally compact group $G$. The measure $\mu$ is called central if $\mu\left(x S x^{-1}\right)=\mu(S)$ for all Borel sets $S$ and all $x \in G$. Central measures are precisely the measures in the center of the measure algebra $M(G)$ (see [2, p. 269]). We shall show that for a connected semisimple Lie group with no nontrivial compact quotient any central measure is a discrete measure supported on the center of the group.

To state our main result in complete generality we need the concept of the maximal connected compact normal subgroup, $C$, of a connected semisimple Lie group $G$. $C$ is determined as follows. Let $\mathfrak{g}$ be the Lie algebra of $G$ and suppose $\mathfrak{g}=\mathrm{g}_{1}+\cdots+\mathfrak{g}_{n}$ where each $\mathrm{g}_{i}$ is a simple ideal. (We refer to [1] for this (p. 122) and in general for all Lie theory facts we use.) We may assume that for $i<k, \mathfrak{g}_{i}$ is a compact Lie algebra while for $i \geqq k, \mathfrak{g}_{i}$ is not compact. Then $C$ is the connected normal subgroup of $G$ whose Lie algebra is $\mathfrak{g}_{1}+\cdots+\mathfrak{g}_{k-1}$.

Our main result is

THEOREM 1. Let $G$ be a connected semisimple Lie group with $Z=$ Center $(G)$. Let $C$ be the maximal connected compact normal subgroup of $G$. Then the support of any finite central measure on $G$ is contained in $Z C$. In particular if $G$ has no nontrivial compact quotient (which is equivalent to $C=\{e\}$ ), then any finite central measure on $G$ is a discrete measure supported on $Z$.

Received by the editors March 26, 1971.

AMS 1970 subject classifications. Primary 22E30, 43A10, 43A80; Secondary 22E15, $43 \mathrm{~A} 20$.

Key words and phrases. Central measures, measures on Lie groups.

1 Research supported by NSF grant GP-22930.

(c) American Mathematical Society 1972 
Proof. Our approach uses some geometric consequences of semisimplicity. We shall define certain subgroups $G^{p}$ such that $\cap G^{p} \subseteq Z C$. These subgroups have the property that the complement, $G-G^{p}$, can be written as a union of finitely many open sets, $U_{j}^{p}$, such that any compact set $F \subseteq U_{j}^{p}$ has countably many disjoint conjugates, $g_{n} F_{n}^{-1}$.

Suppose we have a collection of subgroups and open sets with these properties. Then it is easy to prove that any finite central measure $\mu$ is supported on $Z C$. First note that if a compact set $F$ has countably many disjoint conjugates then $\mu(F)=0$. This follows from $\mu\left(g_{n} F g_{n}^{-1}\right)=\mu(F)$ and $\sum\left|\mu\left(g_{n} F g_{n}^{-1}\right)\right| \leqq|\mu|(G)<\infty$. Thus any compact $F \subseteq U_{j}^{p}$ has $\mu(F)=0$, so by regularity $|\mu|\left(U_{j}^{p}\right)=0$. But

$$
\bigcup_{p} \bigcup_{j} U_{j}^{p}=\bigcup_{p}\left(G-G^{p}\right)=G-\bigcap_{p} G^{p} \supseteq G-Z C .
$$

Thus the support of $\mu$ is contained in $Z C$. If $C=\{e\}$, then $\mu$ is discrete since $Z$ is countable.

We proceed to construct the groups $G^{p}$ and open sets $U_{j}^{p}$. Let Ad: $G \rightarrow$ End $(\mathrm{g})$ be the adjoint representation of $G$. Let $P=\{p \in G: \operatorname{Ad}(p)$ is diagonalizable (over $R$ ) with positive eigenvalues $\}$ and for $p \in P$ set

$$
G^{p}=\{g \in G: \operatorname{Ad}(g p)=\operatorname{Ad}(p g)\} .
$$

First we analyse the complement of a single $G^{p}$. Fix $p \in P$ and fix a basis for $g$ which diagonalizes the matrix for $\operatorname{Ad}(p)$. For any $g \in G$ let $\operatorname{Ad}(g)_{i j}$ be the $i, j$ coordinate in the matrix for $\operatorname{Ad}(g)$ with respect to this fixed basis. Then since $\operatorname{Ad}(p)$ is diagonal we have

$$
\operatorname{Ad}(g p)_{i j}=\operatorname{Ad}(g)_{i j} \operatorname{Ad}(p)_{j j}, \quad \operatorname{Ad}(p g)_{i j}=\operatorname{Ad}(p)_{i i} \operatorname{Ad}(g)_{i j} .
$$

Hence $g \in G^{p}$ if and only if $\operatorname{Ad}(g)_{i j}=0$ for all $(i, j)$ such that $\operatorname{Ad}(p)_{i i} \neq$ $\operatorname{Ad}(p)_{j j}$. So if we set $J_{p}=\left\{(i, j): \operatorname{Ad}(p)_{i i} \neq \operatorname{Ad}(p)_{j j}\right\}$, we can write

$$
G-G^{p}=\bigcup\left\{U_{i j}^{p}:(i, j) \in J_{p}\right\} \quad \text { where } U_{i j}^{p}=\left\{g \in G: \operatorname{Ad}(g)_{i j} \neq 0\right\} .
$$

Note that $U_{i j}^{p}$ is open since $g \mapsto \operatorname{Ad}(g)_{i j}$ is a continuous function.

Now suppose $F \subseteq U_{i j}^{p}$ is compact and let $m, M$ be such that

$$
0<m=\min _{F}\left|\operatorname{Ad}(g)_{i j}\right|<\max _{F}\left|\operatorname{Ad}(g)_{i j}\right|=M .
$$

For $(i, j) \in J_{p}, 0<a=\operatorname{Ad}(p)_{i i} \operatorname{Ad}(p)_{j j}^{-1} \neq 1$. Also

$$
\operatorname{Ad}\left(p^{l} g p^{-l}\right)_{i j}=\operatorname{Ad}(p)_{i i}^{l} \operatorname{Ad}(p)_{j j}^{-l} \operatorname{Ad}(g)_{i j}=a^{l} \operatorname{Ad}(g)_{i j} .
$$

So if we choose $l \in Z$ such that $|a|^{l} m>M$, then the sequence of conjugates of $F$ given by $F_{n}=p^{n l} F p^{-n l}$ is disjoint since

$$
\min _{F_{n+1}}\left|\operatorname{Ad}(g)_{i j}\right|=|a|^{(n+1) l} m>|a|^{n l} M=\max _{F_{n}}\left|\operatorname{Ad}(g)_{i j}\right| .
$$


All that remains is to show that $Z C \supseteq \bigcap_{p} G^{p}$. As a preliminary we show that the group $D=\bigcap_{p} G^{p}$ is normal in $G$. To see this note that $g P g^{-1}=P$ since $\operatorname{Ad}\left(g p g^{-1}\right)=\operatorname{Ad}(g) \operatorname{Ad}(p) \operatorname{Ad}(g)^{-1}$ is diagonalizable if and only if $\operatorname{Ad}(p)$ is diagonalizable and each has the same eigenvalues. Also $G^{g p g^{-1}}=g^{-1} G^{p} g$ because $\operatorname{Ad}\left(g_{0} g p g^{-1}\right)=\operatorname{Ad}\left(g p g^{-1} g_{0}\right)$ if and only if $\operatorname{Ad}\left(g^{-1} g_{0} g p\right)=\operatorname{Ad}\left(p g^{-1} g_{0} g\right)$. Thus

$$
g D g^{-1}=\bigcap g G^{p} g^{-1}=\bigcap G^{g^{-1} p g}=\bigcap G^{p}=D
$$

and so $D$ is normal.

To complete the proof we first consider the case when $G$ is simple and noncompact. Then either $D=G$ or $D=Z$, since clearly $D \supseteq Z$ and $D$ is closed and normal. But the noncompactnes $\bar{s}$ of $G$ implies there exists $p \in G-Z$ with $\operatorname{Ad}(p)$ diagonalizable with positive eigenvalues (take $p=\exp x$ for $x \neq 0$ in the $p$-part of some Cartan decomposition of $g$ $[1$, p. 215]). If $D=G$ then $\operatorname{Ad}(p)$ would commute with $\operatorname{Ad}(G)$ which is impossible since the center of $\operatorname{Ad}(G)$ is trivial. Thus in this case $D=Z$ as desired.

Now pass to the case of the general semisimple $G$ and let $G_{i}$ be the connected normal subgroup with Lie algebra $g_{i}$, where $g=g_{1}+\cdots+g_{n}$ is the decomposition into simple ideals. Then $G=G_{1} \cdots \cdot G_{n}$ since the product on the right is a connected subgroup of $G$ with Lie algebra $\mathfrak{g}$. Also $C=G_{1} \cdot \cdots \cdot G_{k-1}$. Moreover, since $\operatorname{Ad}(g) g_{i} \subseteq g_{i}$ and $\operatorname{Ad}\left(g_{i}\right)\left\lceil\mathfrak{g}_{j}=I\right.$ for $g_{i} \in G_{i}, j \neq i$, it follows that if $p=a_{1} \cdots a_{n}$ with $a_{i} \in G_{i}$, then $p \in P$ if and only if $a_{i} \in P$. From this it is easy to derive that if $d=b_{1} \cdots b_{n}$ with $b_{i} \in G_{i}$ then $d \in D$ if and only if $b_{i} \in D$. Hence $D=D_{1} \cdots \cdot D_{n}$ where $D_{i}=D \cap G_{i}$. Now for $i \geqq k, G_{i}$ is simple and noncompact, so as in the previous case $D_{i}=\operatorname{Center}\left(G_{i}\right)=Z_{i}$. Thus $D \subseteq G_{1} \cdots \cdot G_{k-1} \cdot Z_{k} \cdot \cdots \cdot Z_{n}=C Z$ and we are done.

We remark that $Z C$ cannot be replaced by any smaller group since we can write $Z C=\bigcup_{z \in Z} z C$ and each of the sets $z C$ is the support set of a central measure. Specifically if $m_{C}$ is the (left) Haar measure for $C$, then $m_{C}$ is invariant under the automorphisms of $C$ given by conjugation by elements of $G$, so considered as a measure on $G, m_{C}$ is a finite central measure which is supported on $C$. The measure $\varepsilon_{z} * m_{C}$, where $\varepsilon_{z}$ is the point mass at $z \in Z$, is a central measure whose support is $z C$.

Now we prove a result on the triviality of the center of $L_{1}(H)$. Although this result can be proved using representation theory and direct integral decompositions we believe our proof is more direct. (An alternative proof of the following theorem may also be derived from the results in [4].)

THEOREM 2. Let $H$ be a locally compact group which has a quotient group $G=H / H_{0}$ which is a connected noncompact simple Lie group. Then the center of $L_{1}(H)$ is trivial. 
Proof. In outline the proof proceeds as follows. Let $f$ be in the center of $L_{1}(H)$ and let $m_{0}$ be left Haar measure on $H_{0}$. It will be shown that: (1) The function $|f|^{\prime}\left(z H_{0}\right)=\int_{H_{0}}|f|(z y) d m_{0}(y)$ is in the center of $L_{1}(G)$; (2) the injection of $L_{1}(G)$ into $M(G)$ takes central functions to continuous central measures; (3) if $|f|^{\prime}=0$ then $f=0$. Since $G$ is a noncompact simple Lie group the previous theorem shows that 0 is the only continuous central measure. Hence (1) and (2) imply $|f|^{\prime}=0$. So by (3) it follows that $f=0$ as desired. We now verify (1), (2), and (3).

First we prove (2): if $m_{G}$ is left Haar measure on $G$ then the injection of $L_{1}(G)$ into $M(G)$ given by $f \mapsto f m_{G}$ takes central functions to continuous central measures. The fact that $f m_{G}$ is a continuous measure is clear. Now let $\left\{i_{\alpha}\right\}$ be a two-sided approximate identity for $L_{1}(G)$. Then, identifying $L_{1}(G)$ with its image in $M(G)$ and using the fact that $L_{1}(G)$ is an ideal in $M(G)$, we have, for any $\mu \in M(G)$ and $f$ in the center of $L_{1}(G)$,

$$
\begin{aligned}
j * \mu & =\left(\lim _{\alpha} f * i_{\alpha}\right) * \mu=\lim _{\alpha}\left(f *\left(i_{\alpha} * \mu\right)\right) \\
& =\lim _{\alpha}\left(\left(i_{\alpha} * \mu\right) * f\right)=\lim _{\alpha} i_{\alpha} *(\mu * f)=\mu * f .
\end{aligned}
$$

Hence $f m_{G}$ is in the center of $M(G)$.

We shall need a functional equation which characterizes central functions in $L_{1}(H)$. Since $\mu$ in $M(H)$ is central if and only if $\varepsilon_{x} * \mu=\mu * \varepsilon_{x}$ for all $x \in H$, the previous paragraph, applied to $H$ instead of $G$, shows that $f \in L_{1}(H)$ is central if and only if $\varepsilon_{x} * f=f * \varepsilon_{x}$ for all $x \in H$. The use of the formula for the convolution of a measure and an $L_{1}$-function [2, p. 290], shows this last equality is equivalent to the equation

$$
{ }_{x} f=\Delta_{H}(x) f_{x}, \quad \text { for each } x \in H
$$

where $\Delta_{H}(x)$ is the modular function for $H$ and ${ }_{x} f$ and $f_{x}$ are the left and right translates of $f$ by $x$.

Now we verify (3) and (1). Since we want to relate central functions on $H$ and $G=H / H_{0}$, we compare their modular functions. It is known [3, p. 91] that for $f \in L_{1}(H)$ the formula $f^{\prime}\left(z H_{0}\right)=\int_{H_{0}} f(z y) d m_{0}(y)$ defines $f^{\prime}$ as a function in $L_{1}(G)$ and that the left Haar measures can be appropriately normalized so that $\int_{H} f d m_{H}=\int_{G} f^{\prime} d m_{G}$. From this it is clear that if $|f|^{\prime}=0$ then $f=0$, so (3) holds. Now let $\delta: H \rightarrow \boldsymbol{R}^{+}$be the homomorphism such that

$$
\int_{H_{0}} \varphi\left(x y x^{-1}\right) d m_{0}(y)=\delta(x) \int_{H_{0}} \varphi(y) d m_{0}(y), \quad \varphi \in L_{1}\left(H_{0}\right) .
$$

(Such $\delta$ must exist since the left side is a left invariant integral on $H_{0}$.) 
Then for $f \in L_{1}(H)$

$$
\begin{aligned}
\int_{H} f_{w}(x) d m_{H}(x) & =\int_{G}\left(\int_{H_{0}} f_{w}(z y) d m_{0}(y)\right) d m_{G}\left(z H_{0}\right) \\
& =\int_{G} \delta\left(w^{-1}\right)\left(\int_{H_{0}} f(z w y) d m_{0}(y)\right) d m_{G}\left(z H_{0}\right) \\
& =\Delta_{G}\left(w^{-1}\right) \delta\left(w^{-1}\right) \int_{H} f(x) d m_{H}(x) .
\end{aligned}
$$

So $\Delta_{H}=\Delta_{G} \delta$. (Although $\Delta_{G} \equiv 1$ we shall not need this fact.)

Finally, to show that (1) holds, assume $f$ is a central function in $L_{1}(H)$. Then $|f|$ is also a central function since it satisfies the functional equation $(*)$. Now using $\left({ }^{*}\right)$, the definition of $\delta$, and the formula for $\Delta_{H}$ we see that

$$
\begin{aligned}
{ }_{x H_{0}}|f|^{\prime}\left(z H_{0}\right) & =\int_{H_{0}}|f|(z y) d m_{0}(y)=\int_{H_{0}} \Delta_{H}(x)|f|_{x}(z y) d m_{0}(y) \\
& =\Delta_{H}(x) \delta\left(x^{-1}\right) \int_{H_{0}}|f|(z x y) d m_{0}(y)=\Delta_{G}(x)|f|_{x H_{0}}^{\prime}\left(z H_{0}\right) .
\end{aligned}
$$

Thus $|f|^{\prime}$ is a central function in $L_{1}(G)$, since it satisfies $\left({ }^{*}\right)$ applied to the group $G$. This completes the proof.

\section{REFERENCES}

1. S. Helgason, Differential geometry and symmetric spaces, Pure and Appl. Math., vol. 12, Academic Press, New York, 1962. MR 26 \#2986.

2. E. Hewitt and K. Ross, Abstract harmonic analysis. Vol. 1 : Structure of topological groups. Integration theory, group representations, Die Grundlehren der math. Wissenschaften, Band 115, Academic Press, New York; Springer-Verlag, Berlin, 1963. MR 28 \#158.

3. - Abstract harmonic analysis. Vol. 2: Structure and analysis for compact groups, analysis on locally compact Abelian groups, Die Grundlehren der math. Wissenschaften, Band 152, Springer-Verlag, New York, 1970. MR 41 \#7378.

4. R. Mozak, Central functions in group algebras, Proc. Amer. Math. Soc. 29 (1971), 613-616.

Department of Mathematics, Massachusetts institute of Technology, CAMbridge, Massachusetts 02139

Department of Mathematics, Tufts University, Medford, Massachusetts 02155

Current address (Ragozin): Department of Mathematics, University of Washington, Seattle, Washington 98195 